\title{
Rationale for the Early Use of Sodium-Glucose Cotransporter-2 Inhibitors in Patients with Type 2 Diabetes
}

Yehuda Handelsman (D)

Received: June 7, 2019 / Published online: August 23, 2019

(c) The Author(s) 2019 provide the most benefit toward glycemic control and CV and renal risk. Thus, current treatment recommendations include the early addition of SGLT-2 inhibitor therapy, not only in patients with established CVD, HF, and/or CKD but also in the general population of patients with T2D.

Funding: AstraZeneca.

Keywords: Canagliflozin; Cardiovascular effects; Dapagliflozin; Early treatment; Empagliflozin; Ertugliflozin; Glycemic control; Renal effects; Sodium-glucose cotransporter-2 inhibitors; Type 2 diabetes

\section{PLAIN LANGUAGE SUMMARY}

People with type 2 diabetes (T2D) are at risk of developing complications, including macrovascular [cardiovascular disease (CVD), strokes, and heart failure (HF)] and microvascular [chronic kidney disease (CKD) and damage to the eyes and nerves] conditions. Reduction of blood sugar (glucose) levels (glycemic control) can prevent or halt these complications. Sodium-glucose cotransporter-2 (SGLT-2) inhibitors are a class of glucose-lowering drugs that improve glycemic control, reduce CV (cardiovascular) risk factors such as being overweight and having high blood pressure, and have a low risk of hypoglycemia (low blood sugar levels).

Y. Handelsman $(\bowtie)$

Metabolic Institute of America, Tarzana, CA, USA e-mail: yhandelsman@gmail.com 
Health care providers who treat people with T2D sometimes add SGLT-2 inhibitors after initial treatment with metformin alone. Recently, clinical trials and real-world studies in patients with T2D have shown that SGLT-2 inhibitors (empagliflozin, canagliflozin, and dapagliflozin) reduced the risk of $\mathrm{CV}$ events, $\mathrm{CV}$ death, and hospitalization for HF and reduced worsening of kidney disease. Although the reduction in CV events with SGLT-2 inhibitor treatment was greatest for patients who already had CVD, the risk was also reduced in those without CVD. Reductions in death from $\mathrm{CV}$ causes or hospitalization for $\mathrm{HF}$ and kidney events were similar for patients with or without CVD. Some updated treatment guidelines for T2D, therefore, recommend early use of SGLT-2 inhibitors in patients with T2D, not only those with established CVD. This review describes the reasons for starting early treatment with SGLT-2 inhibitors when the potential benefit may be greatest, providing protection against $\mathrm{CV}$ events, hospitalization for $\mathrm{HF}$, and progression of CKD.

\section{INTRODUCTION}

The burden of diabetes in the United States is considerable and growing. An estimated 30.3 million Americans had diabetes in 2015 (9.4\% of the population) [1], and projections suggest a prevalence of $\sim 55$ million by 2030 [2], with $\sim 90-95 \%$ of these individuals having type 2 diabetes (T2D) [1].

A high proportion of diabetes-related disease burden and cost can be attributed to comorbidities and complications [3], including congestive heart failure (HF), atherosclerotic cardiovascular disease (CVD), peripheral vascular disease, chronic kidney disease (CKD), neuropathy, and retinopathy $[1,3]$. Although the rate of diabetes-related complications is decreasing, the increasing prevalence of T2D means the number of individuals who develop diabetes-related illnesses remains substantial [4]. There is, therefore, an urgent need to reduce T2D-associated morbidity and mortality through the effective management of glycemia, $\mathrm{CV}$ risk factors, and other risk factors of chronic disease.

Early intervention to achieve glycemic control and reduce $\mathrm{CV}$ and renal risk is important, because a high proportion of patients with T2D already have risk factors before diagnosis [5]. In the National Health and Nutrition Examination Survey (NHANES), $61.9 \%$ of patients with undiagnosed T2D had hypertension, $82.6 \%$ had hypercholesterolemia, $56.8 \%$ had obesity, and an additional $29.5 \%$ were overweight [5]. Current American Diabetes Association (ADA) and American Association of Clinical Endocrinologists/American College of Endocrinology (AACE/ACE) guidelines recommend regular assessment of $\mathrm{CV}$ risk factors in patients with diabetes and treatment of any modifiable risk factors outside the normal range [6,7]. Both guidelines also emphasize the importance of patient-centered care, in which treatment is tailored to patients' individual preferences and needs, including effects on CV outcomes, risk factors, glycemic control, body weight, and renal function $[7,8]$.

Agents from two classes of glucose-lowering therapies, sodium-glucose cotransporter (SGLT)-2 inhibitors and glucagon-like peptide-1 receptor agonists (GLP-1RAs), have been shown to significantly reduce the incidence of $\mathrm{CV}$ events in patients with T2D. In large-scale CV outcomes trials (CVOTs), the SGLT-2 inhibitors empagliflozin, canagliflozin, and dapagliflozin [9-11], and the GLP-1RAs liraglutide, semaglutide, dulaglutide, and albiglutide [12-15], significantly reduced the risk of $\mathrm{CV}$ events. In addition, although the GLP-1RA exenatide once weekly did not show superiority to placebo in the overall population of the Exenatide Study of Cardiovascular Event Lowering (EXSCEL) CVOT [16], a follow-up analysis showed CV events were significantly reduced in patients with established CVD [17]. While most CVOTs showed reduction of $\mathrm{CV}$ events in patients with CVD at baseline [11-14, 17, 18], some also demonstrated this in patients without established CVD $[9,10,15]$.

This review describes the rationale for early initiation of SGLT-2 inhibitors, when the potential to modify outcomes may be greatest, 
to provide protection against $\mathrm{CV}$ events and prevent the development of $\mathrm{HF}, \mathrm{CKD}$, and microvascular complications.

This article is based on previously conducted studies and does not include any new studies with human participants or animals performed by the author.

\section{RATIONALE FOR EARLY RISK MANAGEMENT}

Evidence from clinical trials indicates that early intervention and achievement of glycemic control reduces the long-term risk of microvascular and macrovascular complications in T2D. Two landmark studies, the UK Prospective Diabetes Study (UKPDS) in patients with newly diagnosed T2D and the Action in Diabetes and Vascular Disease: Preterax and Diamicron Modified Release Controlled Evaluation (ADVANCE) study in patients with a mean T2D duration of $\sim 8$ years at baseline, demonstrated that intensive glycemic control [treatment with a sulfonylurea or insulin, or metformin for patients with body weight $>120 \%$ of ideal, in the UKPDS and treatment to a glycated hemoglobin A1c (HbA1c) level of $<6.5 \%$ in ADVANCE] not only reduced the occurrence of microvascular complications but also significantly reduced the long-term incidence of macrovascular events, including myocardial infarction (MI) [19, 20].

In the Intensified Multifactorial Intervention in Patients With Type 2 Diabetes and Microalbuminuria (Steno-2) study (median diabetes duration of 4-6 years at baseline), intensive multifactorial treatment that targeted several CV risk factors was associated with significant reductions in the risk of mortality (45\%; $p=0.005), \mathrm{CV}$ events (45\%; $p<0.001)$, and microvascular complications (range, 33\%-48\%) compared with conventional therapy over 21.2 years of follow-up [21]. However, in the Action to Control Cardiovascular Risk in Diabetes (ACCORD) study [22] and the Veterans Affairs Diabetes Trial (VADT) [23], in which the duration of diabetes at baseline was longer (median of 10 years and mean of 11.5 years, respectively), the results are less clear. Although these studies showed no significant effect of intensive glycemic control on CV complications [22, 23], the intensive therapy arm of the ACCORD study was stopped early (after 3.5 years) because of a significant increase in mortality [22]. This was despite a significantly reduced risk of nonfatal MI with intensive therapy [22].

The ADA, American Heart Association (AHA), and American College of Cardiology (ACC) reviewed these apparently contradictory data and determined that the differences were at least partly due to patient characteristics [24]. Patients most likely to have reduced CVD risk with intensive glycemic control were those with a shorter T2D duration who had not yet developed atherosclerosis, whereas, in older or frail patients, those with a long duration of T2D or patients with advanced atherosclerotic disease, the risks associated with intensive glycemic control may exceed its benefits [24]. In a post hoc analysis of ACCORD, the greatest risk of mortality with intensive therapy appeared to be among patients who continued to have an HbA1c level of $>7.0 \%$ [25], highlighting the need for personalized medicine and individualized treatment goals, as recommended by treatment guidelines $[6,7]$. These findings were supported by a subsequent analysis of VADT, which found that intensive glycemic control reduced the risk of $\mathrm{CV}$ events in patients with a T2D duration of $<15$ years, but was associated with increased risk in those with a disease duration of $\geq 15$ years and was potentially harmful in those with a disease duration of $\geq 20$ years [26].

A 10-year post-trial follow-up of UKPDS found a significant reduction in the risk of $\mathrm{CV}$ events in patients with newly diagnosed T2D who had received intensive glycemic control, but only after enough time had passed to demonstrate an effect on these outcomes [19]. This study demonstrated a legacy effect of early intensive therapy because the difference in the $\mathrm{CV}$ event rate 10 years post-trial was apparent even though there was no longer a difference in HbA1c levels between the intensive and conventional therapy groups [19].

A similar legacy effect was seen in patients with type 1 diabetes who had received intensive or conventional therapy for 6.5 years in the 
Diabetes Control and Complications Trial (DCCT) [27]. Thirty-year post-trial follow-up data showed that, among young patients who had not yet developed CVD, early intensive diabetes therapy significantly reduced the later risk of a CV event by $30 \%$ compared with conventional therapy $(p=0.016)$ [27].

Real-world evidence has also shown a legacy effect of early intensive glycemic control in patients with T2D. The Diabetes \& Aging Study found that, after 13 years' follow-up, patients who achieved an HbA1c of $<6.5 \%$ during the first year after diagnosis had a lower risk of microvascular and macrovascular complications than those with $\mathrm{HbA} 1 \mathrm{c} \geq 6.5 \%$ and a lower mortality risk than those with $\mathrm{HbA} 1 \mathrm{c} \geq 7.0 \%$ during the first year [28].

Early use of combination therapy in patients with newly diagnosed T2D has also been shown to reduce the progression of atherosclerosis, in addition to improving glycemic control, compared with conventional therapy $[29,30]$. In the Efficacy and Durability of Initial Combination Therapy for Type 2 Diabetes (EDICT) study, firstline treatment with exenatide, pioglitazone, and metformin was associated with a greater reduction in HbA1c and more patients achieving glycemic targets after 2 years compared with initial treatment with metformin and sequential addition of a sulfonylurea and insulin glargine [29]. The greater reduction in HbA1c was maintained at 6 years, and the early combination therapy group also had significantly reduced carotid intimal media thickness compared with the conventional therapy group [30].

These studies demonstrate that early achievement of glycemic targets has the greatest effect on macrovascular complications, with a legacy effect even if intensive glycemic control is not maintained. However, it also raises the question whether continued intensive therapy would provide much earlier benefits beyond the legacy effect.

\section{MECHANISM OF GLYCEMIC CONTROL OF SGLT-2 INHIBITORS}

SGLT-2 inhibitors act by insulin-independent mechanisms to improve glycemic control and
CV risk factors in patients with T2D. SGLT-2 inhibitors reduce reabsorption of glucose from the proximal tubule of the kidneys, which in turn increases glucosuria [31]. Because inhibition of SGLT-2 also reduces sodium reabsorption, SGLT-2 inhibitors have a natriuretic effect, which may partially explain the observed reduction in blood pressure (BP) [32]. These reductions in $\mathrm{BP}$ are not accompanied by increases in heart rate, indicating a lack of reflex sympathetic nervous system activation [33]. The natriuretic effects of SGLT-2 inhibitors, which may lead to reductions in plasma volume and cardiac preload, also occur without activation of the renin-angiotensin-aldosterone system [34]. Although the specific effects of SGLT-2 inhibitors on intrarenal hemodynamics are unclear, changes in tubuloglomerular feedback may be involved in neurohormonal stimulation and fluid and electrolyte homeostasis, and the net effect of SGLT-2 inhibition in the diabetic kidney appears to be protective and associated with preservation of renal function [34].

SGLT-2 inhibitors are also associated with increased lipolysis, with an early shift in substrate utilization from carbohydrates to fats [32]. Coupled with increased glucosuria and other as yet unknown mechanisms, such as a reduction in sympathetic nervous system activity [35], the net effect of SGLT-2 inhibitor treatment is a reduction in both body weight and fat mass [32]. Studies have also shown decreases in hepatic fat [36, 37] and fibrosis [37-39] with SGLT-2 inhibitors in patients with T2D and nonalcoholic fatty liver disease, and reduced epicardial fat accumulation in patients with T2D [40]. Reductions in body weight and $\mathrm{BP}$ (independent of glucosuria) have also been observed with SGLT-2 inhibitor therapy in patients with T2D and CKD [estimated glomerular filtration rate (eGFR) $30-59 \mathrm{~mL} /$ $\mathrm{min} / 1.73 \mathrm{~m}^{2}$ ] in whom there is reduced glucosuria compared to patients with normal or mildly impaired renal function [41].

Because the SGLT-2 inhibitor mechanism of action is independent of insulin, and differs from those of other classes of glucose-lowering therapy, which typically affect beta-cell function, hepatic glucose production, or glucose uptake by the muscles, SGLT-2 inhibitors can 
potentially act synergistically with other agents [32]. Unlike other glucose-lowering therapy classes, efficacy with SGLT-2 inhibitors does not decline with worsening beta-cell function [32]. Furthermore, SGLT-2 inhibitors are associated with a low risk of hypoglycemia [31].

\section{CARDIOVASCULAR AND RENAL EFFECTS OF SGLT-2 INHIBITORS}

\section{Randomized Controlled Trials}

The effects of SGLT-2 inhibitors on CV outcomes have been assessed in several randomized, placebo-controlled trials [9-11] and in real-world studies [42-44]. In addition, ertugliflozin is currently being investigated in a largescale CVOT [Evaluation of Ertugliflozin Efficacy and Safety CV Outcomes Trial (VERTIS-CV); NCT01986881] [45], with study completion expected in September 2019.

In the completed CVOTs, the Empagliflozin Cardiovascular Outcome Event Trial in Type 2 Diabetes Mellitus Patients (EMPA-REG OUTCOME) included patients with established CVD (>99\% of patients) and almost no patients $(<1 \%)$ with multiple CVD risk factors [11], while the Canagliflozin Cardiovascular Assessment Study (CANVAS) [9] and the Dapagliflozin Effect on Cardiovascular Events-Thrombolysis in Myocardial Infarction 58 (DECLARE-TIMI 58) study [10] also included patients with multiple CVD risk factors (34\% and $59 \%$ of patients, respectively).

In these studies, empagliflozin and canagliflozin were associated with a $14 \%$ reduction in the risk of major adverse $\mathrm{CV}$ events (MACE) compared with placebo $[9,11]$. The risk of MACE was not significantly lower with dapagliflozin versus placebo; however, dapagliflozin significantly reduced the risk of the coprimary composite end point of CV death or hospitalization for HF by $17 \%$ [10].

SGLT-2 inhibitors were also associated with reductions in the risks of $\mathrm{HF}$ and mortality outcomes compared with placebo in these studies [9-11]. In EMPA-REG, significant reductions in the risk of CV mortality (38\%), allcause mortality $(32 \%)$, and hospitalization for HF (35\%) were observed with empagliflozin compared with placebo [11]. Although the risk of the individual end point of hospitalization for HF was lower with canagliflozin versus placebo in CANVAS, these results were not considered statistically significant because hypothesis testing was not performed based on a prespecified hierarchy of secondary end points $[9,10]$. However, in DECLARE, dapagliflozin had dual primary outcomes and, although it did not demonstrate the superiority of MACE reduction, the reduction in hospitalization for HF and CV Mortality was statistically significant [10].

Differences in study design, including exclusion of patients with a creatinine clearance of $<60 \mathrm{~mL} / \mathrm{min}$ and inclusion of a large proportion of patients without established CVD, may have affected the findings in DECLARE [10]. A prespecified analysis of patients with prior MI in DECLARE found significant reductions in the risk of MACE, as well as CV mortality or hospitalization for HF [46]. A metaanalysis of CANVAS, DECLARE, and EMPA-REG found that, while the risk of MACE was reduced by $11 \%$ in the overall population, the reduction was primarily in the subgroup with established $\mathrm{CVD}$, and there was no significantly reduced risk in the subgroup without CVD [47]. However, the risks of $\mathrm{CV}$ death or hospitalization for $\mathrm{HF}$ and renal outcomes were reduced in both the subgroup without established CVD and the subgroup with established CVD, as well as the overall population.

While all SGLT-2 inhibitor CVOTs showed a reduction in the risk of renal outcomes with SGLT-2 inhibitors versus that with placebo [10, 18, 48, 49], in CANVAS and DECLARE, it was also seen in patients without CVD at baseline $[10,48]$. Canagliflozin was associated with a reduction in the risk of albuminuria progression by $27 \%$ overall and by $31 \%$ in the subgroup without CVD [48]. Despite the DECLARE population having a mean eGFR of $85.2 \mathrm{~mL} / \mathrm{min} /$ $1.73 \mathrm{~m}^{2}$ and $48 \%$ of patients having eGFR $\geq 90 \mathrm{~mL} / \mathrm{min} / 1.73 \mathrm{~m}^{2}$ at baseline, dapagliflozin was associated with a reduction in the risk of the composite outcome of $40 \%$ decrease in eGFR, end-stage renal disease (ESRD), or death from renal causes by $47 \%$ overall and by $49 \%$ in the subgroup without CVD [10]. 
Reductions in individual components of this end point (eGFR reduction of $\geq 40 \%$ to $<60 \mathrm{~mL} / \mathrm{min} / 1.73 \mathrm{~m}^{2}$ and ESRD) were also observed with dapagliflozin $(p<0.05$ vs. placebo) [50]. In addition, dapagliflozin, given with or without saxagliptin, was found to significantly reduce albuminuria in a study in patients with T2D, moderate-to-severe CKD, and micro- or macroalbuminuria [51]. The impact of the SGLT-2 inhibitor class on the kidney was further shown in Canagliflozin and Renal Events in Diabetes with Established Nephropathy Clinical Evaluation (CREDENCE), a dedicated renal outcomes trial comparing canagliflozin with placebo given in addition to the standard of care in patients with T2D and CKD (eGFR $\geq 30$ to $<90 \mathrm{~mL} / \mathrm{min} / 1.73 \mathrm{~m}^{2}$ and albuminuria) [52]. Canagliflozin reduced the relative risk of the primary composite end point of ESRD, doubling the serum creatinine level, or of renal or CV death by $30 \%(p=0.00001)$; relative risks of the individual components of ESRD and doubled serum creatinine were also significantly reduced [53]. Significant reductions were also seen in the relative risks of the secondary end points of CV death or hospitalization for $\mathrm{HF}$ (31\% reduction); CV death, MI, or stroke (20\%); hospitalization for HF (39\%); and ESRD, doubled serum creatinine, or renal death (34\%) [53].

\section{Real-World Evidence}

Although randomized controlled trials are necessary to demonstrate the efficacy of different treatments, their highly selected patient populations (especially the population with established CVD) can limit the generalizability of results to clinical practice populations [54, 55]. Given the low number of CV events among patients without established CVD, these studies would need to include much larger numbers of patients to be sufficiently powered to confirm improved CV outcomes in this subgroup. Conducting real-world observational studies allows the study of outcomes in large patient populations, and may also provide supplemental evidence of treatment efficacy that potentially increases the applicability of treatment recommendations to clinical practice $[54,55]$.

The Comparative Effectiveness of CV Outcomes in New Users of SGLT-2 Inhibitors (CVD-REAL) study compared CV outcomes in $>300,000$ propensity-matched patients with T2D who newly initiated SGLT-2 inhibitors or another form of glucose-lowering therapy in the United States, United Kingdom, Denmark, Germany, Norway, and Sweden [42]. In this study, in which $13 \%$ of patients had established CVD and $<3 \%$ had CKD, SGLT-2 inhibitor therapy was associated with significantly lower risks of all-cause mortality, hospitalization for $\mathrm{HF}$, and the composite outcome of all-cause mortality or hospitalization for HF compared with other glucose-lowering therapies (Fig. 1) [42]. These findings were consistent across the unadjusted intent-to-treat analysis (Fig. 1a) and the on-treatment analysis after adjustment for age, sex, frailty, hypertension, obesity/body mass index, duration of T2D, use of antihypertensive agents, and history of $\mathrm{HF}, \mathrm{MI}$, and atrial fibrillation (Fig. 1b) [42]. In an analysis of the Nordic subgroup of CVD-REAL participants (in Norway, Denmark, and Sweden), there were significant decreases in risks of CV mortality (by $47 \%$ ) and major CV events (by 22\%) with SGLT-2 inhibitors versus those with other glucose-lowering therapies $(p<0.0001)$ [43]. Similarly, in the CVD-REAL 2 study of $>400,000$ propensity-matched patients with T2D in the Asia-Pacific region, Middle East, and North America, who newly initiated an SGLT-2 inhibitor or other glucose-lowering therapy, in which $\sim 27 \%$ had established CVD and $<2 \%$ had CKD, SGLT-2 inhibitors were associated with a reduced risk of all-cause mortality, hospitalization for HF, the composite of all-cause mortality or hospitalization for HF, MI, and stroke, compared with other glucose-lowering therapies ( $p \leq 0.001$ for all) [44]. Of note, the risk of these outcomes was reduced not only in patients with established CVD but also in those without CVD at baseline [44].

A real-world meta-analysis of four US databases (OBSERVE-4D) assessed hospitalization for HF outcomes among $>700,000$ patients with T2D who newly initiated canagliflozin, other SGLT-2 inhibitors, or other non-SGLT-2 


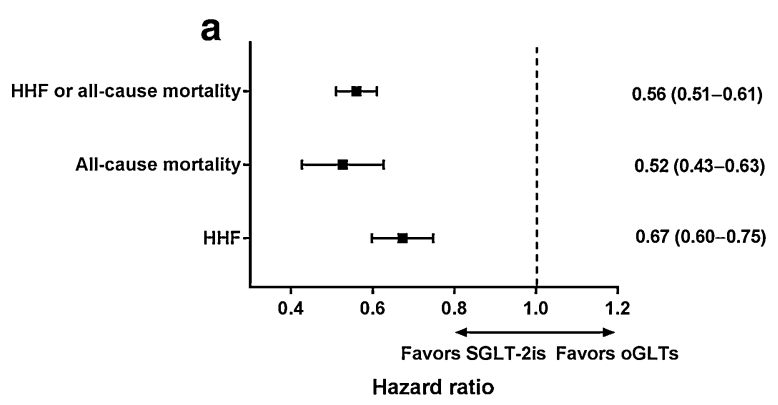

Fig. 1 Cardiovascular outcomes in real-world patients receiving SGLT-2is or oGLTs in the CVD-REAL study [42]. a Unadjusted intent-to-treat analysis. b On-treatment analysis adjusted for history of heart failure; age; sex; frailty; history of myocardial infarction; history of atrial fibrillation; hypertension; obesity/body mass index; duration of type 2 diabetes; and use of angiotensin-converting

inhibitor therapy, 30\% of whom had established CVD [56]. In OBSERVE-4D, there was a significant reduction in the risk of hospitalization for HF in the overall population with newly initiated canagliflozin versus non-SGLT-2 inhibitor therapy (by 61\%) and with newly initiated other SGLT-2 inhibitors versus non-SGLT-2 inhibitor therapy (by 57\%); these reductions were similar to those observed in the subgroup with established CVD [56]. However, no difference was observed with canagliflozin versus other SGLT-2 inhibitors [56].

Initial results from the Empagliflozin Comparative Effectiveness and Safety (EMPRISE) study also showed that empagliflozin and other SGLT-2 inhibitors reduced hospitalization for HF in patients with and without established CVD compared with dipeptidyl peptidase-4 (DPP-4) inhibitors [57]. Analysis of propensity score-matched patient pairs found that empagliflozin reduced the risk of hospitalization for $\mathrm{HF}$ as the primary discharge diagnosis and as any discharge diagnosis by $50 \%$ and $49 \%$, respectively, compared with sitagliptin, although the number of events was low [57]. Compared with all DPP-4 inhibitors, empagliflozin reduced the risk of HF as the primary or any-position discharge diagnosis by $51 \%$ and $44 \%$, respectively [57]. Similarly, comparison of the two drug classes indicated that, compared with DPP-4 inhibitors, SGLT-2 inhibitors were

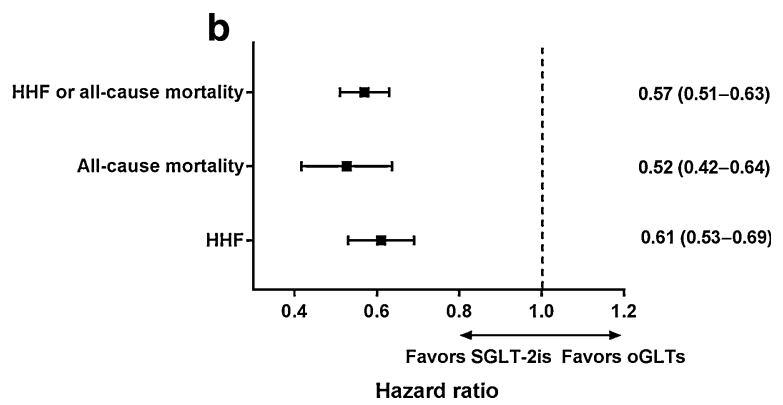

enzyme inhibitors or angiotensin receptor blockers, alphaor beta-blockers, calcium-channel blockers, loop diuretics, or thiazide diuretics. Error bars $95 \%$ confidence interval. $H H F$ hospitalization for heart failure, SGLT-2is sodiumglucose cotransporter-2 inhibitors, $o G L T s$ other glucoselowering therapies

associated with 58\% and 30\% reductions in the risk of primary and any-position hospital discharge diagnosis of HF, respectively [57].

\section{MECHANISMS OF CV AND RENAL PROTECTION WITH SGLT-2 INHIBITORS}

As described in the section "Mechanism of glycemic control of SGLT-2 inhibitors", the effects of glycemic control on CV outcomes can take years to manifest [19]; therefore, the observed effects of SGLT-2 inhibitors in the CVOTs and the CVD-REAL study are likely to result from mechanisms beyond glycemic control [33]. The cardioprotective and renoprotective effects of SGLT-2 inhibitors in patients with T2D are likely multifactorial and encompass additive effects on glycemia and CV risk factors (including BP and body weight) [58, 59]; there are potentially other pathophysiologic mechanisms of atherosclerosis (Table 1) [58]. The effect of SGLT-2 inhibitors on renal function also plays an important role in $\mathrm{CV}$ risk reduction.

There is emerging evidence that, in addition to their effects on CV risk factors, SGLT-2 inhibitors may have other direct effects on $\mathrm{CV}$ function. Because of their natriuretic and diuretic effects, SGLT-2 inhibitors reduce plasma volume and therefore lower cardiac 
Table 1 Change from baseline in cardiovascular risk factors with SGLT-2 inhibitors [58]

\begin{tabular}{ll}
\hline Cardiovascular risk factor & $\begin{array}{l}\text { Change from baseline } \\
(\mathbf{9 5 \%} \mathbf{C I})^{\mathrm{a}}\end{array}$ \\
\hline Blood pressure (mm Hg) & \\
Systolic & $-2.46(-2.86,-2.06)$ \\
Diastolic & $-1.46(-1.82,-1.09)$ \\
Lipid levels (mg/dL) & $0.77(0.33,1.21)$ \\
Total cholesterol & $3.89(3.23,4.56)$ \\
HDL cholesterol & $-2.08(-2.51,-1.64)$ \\
Triglycerides & $0.02(0.01,0.03)$ \\
Lipid levels, mmol/L & $0.10(0.08,0.12)$ \\
Total cholesterol & $-0.02(-0.03,-0.02)$ \\
HDL cholesterol & \\
Triglycerides & $-2.40(-2.68,-2.11)$ \\
Glycemic measures & \\
Fasting blood glucose \\
(mg/dL)
\end{tabular}

Data are from a meta-analysis of 43 controlled trials (14 trials with canagliflozin, 22 with dapagliflozin, 4 with empagliflozin, 2 with remogliflozin, and 1 with ipragliflozin) with a treatment duration range of 4-208 weeks $H b A 1 c$ glycated hemoglobin Alc, CI confidence interval, eGFR estimated glomerular filtration rate, $H D L$ highdensity lipoprotein, $S G L T$ sodium-glucose cotransporter ${ }^{a}$ Blood pressure data are from the overall meta-analysis; all other data are the results of the leave-one-out sensitivity analysis. Results of the leave-one-out sensitivity analyses were similar to those of the primary analysis across all studies and parameters preload [34]. Unloading the heart by this mechanism may explain why SGLT-2 inhibitors have beneficial effects on left ventricular diastolic function, as well as on left ventricular mass $[60,61]$. The reduction in plasma volume with SGLT-2 inhibitors does not appear to be associated with a reflex increase in sympathetic activity [60]. Whether SGLT-2 inhibitors suppress abnormal sympathetic activity, thereby providing patients with diabetes some protection against arrhythmias in the acute setting, is currently being investigated [62].

Another potential mechanism of benefit for SGLT-2 inhibitors in CVD is through mediation of improvements in endothelial function $[63,64]$. The Dapagliflozin Effectiveness on Vascular Endothelial Function and Glycemic Control in T2DM (DEFENCE) study found that treatment with dapagliflozin for 16 weeks significantly improved flow-mediated dilation in the brachial artery compared with metformin [64]. Patients in this study had early T2D (mean duration, $\sim 6$ years) and good glycemic control (mean A1C, < 7\%) [64].

SGLT-2 inhibitor therapy is also associated with an increase in ketone bodies, which results in the cardiac uptake and oxidization of $\beta$-hydroxybutyrate rather than fatty acids [65]. This may promote increased hepatic synthesis of ketones (including $\beta$-hydroxybutyrate) that can be used as an alternative cardiac fuel, potentially providing a more efficient energy source than either glucose or fatty acids [66].

The beneficial effects of SGLT-2 inhibitors in HF outcomes may result from their ability to inhibit sodium-hydrogen exchangers in the heart and kidneys, which could potentially increase the natriuretic effects of other agents routinely administered to patients with HF (e.g., diuretics and mineralocorticoid receptor antagonists) [67]. This may attenuate cardiomyocyte injury and prevent the onset of left ventricular hypertrophy and ultimately HF [67].

Preliminary studies suggest SGLT-2 inhibitors reduce epicardial fat [68], and may also exhibit antifibrotic effects in myocardial and pericardial cells [69-72]. In a study of postinfarction rats, dapagliflozin administration led to decreases in myofibroblast infiltration and collagen deposition that were independent of the 
glucose-lowering effects [70]. At the cellular level, other mechanisms are likely also involved in the renoprotective and cardioprotective effects of SGLT-2 inhibitors, including antiinflammatory and anti-oxidative effects [73], although further research is required to elucidate these mechanisms.

\section{SAFETY CONSIDERATIONS FOR SGLT-2 INHIBITOR THERAPY}

SGLT-2 inhibitors are generally well tolerated and do not increase the risk of hypoglycemia when used with metformin, GLP-1RAs, DPP-4 inhibitors, or thiazolidinediones $[74,75]$. The most common adverse events with SGLT-2 inhibitors are genital mycotic infections $[74,76-79]$. These occur in up to $10 \%$ of patients and in both men and women, although the incidence is lower among circumcised men $[76,78]$. Genital mycotic infections are usually of mild to moderate intensity and can be mitigated through hygiene and the occasional use of antifungal agents. However, an alternative to SGLT-2 inhibitor therapy may be preferable for patients with a history of multiple yeast infections.

Volume depletion-related adverse events, including hypotension and dizziness, have also been reported with SGLT-2 inhibitor therapy [76-79]. Volume depletion occurs more frequently among patients who are older, have a longer duration of T2D, and have eGFR $<60 \mathrm{~mL} / \mathrm{min} / 1.73 \mathrm{~m}^{2}$, and those receiving a concomitant diuretic, angiotensinconverting enzyme inhibitor, or angiotensin receptor blocker therapy [76-79]. Before starting treatment with SGLT-2 inhibitors, volume status should be assessed and hypovolemia corrected. Increasing fluid intake and reducing or discontinuing diuretic treatment can reduce the risk of volume depletion-related events.

Serious, but rare, adverse events associated with SGLT-2 inhibitor therapy include diabetic ketoacidosis (DKA), amputations, fractures, and Fournier's gangrene [76-79]. The risk of such events can be reduced by assessing patients for risk factors before starting treatment with SGLT-2 inhibitors, and monitoring for, and educating patients about, the signs and symptoms of these events during treatment.

DKA primarily occurs in patients who are insulin-deficient, and is generally not seen in those with earlier stage T2D [80]. Patients taking SGLT-2 inhibitors who develop DKA may have normal or less elevated than anticipated blood glucose levels because of the reduced threshold for glucose excretion with this drug class [80]. Patients should be evaluated for predisposing factors for DKA before starting treatment with an SGLT-2 inhibitor [76-80]. To reduce the risk of DKA, SGLT-2 inhibitor therapy should be temporarily discontinued 1-2 days before elective surgery, and before extreme physical activity such as marathon running, and stopped immediately in patients with sepsis or undergoing emergency surgery [80]. In addition, excessive alcohol consumption and ketogenic or very low carbohydrate diets should be avoided [80].

An increased risk of lower limb amputations was seen with canagliflozin in the CANVAS program but not with other SGLT-2 inhibitors in CVOTs [9-11]. However, while amputations were more frequent with canagliflozin versus placebo in the CANVAS program, this was not observed in the CREDENCE trial $[9,53]$. Amputations were more common among patients with a prior history of amputations and among those with severe vascular disease or neuropathy [76, 81]. SGLT-2 inhibitor therapy should be avoided in patients considered to be at increased risk of lower limb amputation, and discontinued in patients who develop ulcers and infections of the lower limbs [76].

Fractures were previously a concern with SGLT-2 inhibitor therapy, but long-term data do not suggest an increased risk [82]. However, factors that may increase a patient's risk for fracture should be considered when prescribing an SGLT-2 inhibitor therapy [76].

Fournier's gangrene is a rare but potentially life-threatening complication of SGLT-2 inhibitor therapy. This adverse event should be managed with broad-spectrum antibacterial agents and surgical debridement as necessary; SGLT-2 inhibitor therapy should be discontinued [76-79]. 
Although serious adverse events have been reported with SGLT-2 inhibitor therapy, the risk should be weighed against the potential benefits of reduced $\mathrm{CV}$ and renal complications. Furthermore, the risk of adverse events is likely to be lower among patients with earlier-stage T2D than that reported in clinical trials and real-world studies, because those data were obtained from across the patient population, including older patients and those with longer duration or greater severity of T2D [80].

\section{PLACE OF SGLT-2 INHIBITORS IN EARLY DIABETES THERAPY}

Current ADA guidelines recommend monotherapy with metformin in combination with lifestyle management as first-line therapy from the time of T2D diagnosis to achieve glycemic control [8]. AACE/ACE guidelines also recommend that pharmacologic treatment be started together with lifestyle management following diagnosis [7]. Patients with an $\mathrm{HbA} 1 \mathrm{c}<7.5 \%$ at diagnosis should receive monotherapy, with metformin being the preferred first-line treatment, although other agents can also be used [7]. For patients whose $\mathrm{HbA} 1 \mathrm{c}$ is $\geq 7.5 \%$ at diagnosis, AACE/ACE guidelines recommend starting on dual therapy with metformin and another class of glucoselowering therapy, with GLP-1RAs and SGLT-2 inhibitors preferred [7]. For patients with HbA1c $>1.5 \%$ above goal at diagnosis, the ADA and European Association for the Study of Diabetes (EASD) guidelines recommend first-line treatment with a dual combination $[8,83]$. The ADA and EASD recommend an SGLT-2 inhibitor as the first post-metformin treatment in patients with established CVD, congestive HF, or CKD $[8,83]$. The ACC/AHA primary prevention guidelines also recommend SGLT-2 inhibitor or GLP-1RA therapy after first-line metformin to reduce $\mathrm{CV}$ risk in patients with T2D and additional CV risk factors [84].

The AACE/ACE and ADA guidelines recommend that patients should be initially assessed every 3 months, and additional glucose-lowering treatments should be added as needed at each assessment to meet glycemic targets $[7,8]$.
For add-on therapy in patients without CVD, the ADA guidelines state that treatment choice should be guided by patient needs, including avoidance of adverse effects (e.g., hypoglycemia and body weight gain), cost considerations, or other needs [8], while the AACE/ACE guidelines recommend a hierarchy of use for add-on glucose-lowering therapy based on safety and efficacy and consideration of the properties of each agent for individual patients [7]. The AACE/ACE guidelines also recommend that GLP-1RAs or SGLT-2 inhibitors with proven CV benefits should be prescribed to patients with CVD regardless of glucose level [7]. The ADA/EASD consensus statement not only recommends SGLT-2 inhibitors in patients with HF or a high risk of HF but also recommends that their use be considered in patients with CKD (with or without $(\mathrm{VD})$ to prevent or reduce progression of CKD [83], although there is some debate regarding the quality of evidence for these recommendations. In patients with $\mathrm{T} 2 \mathrm{D}$ and overweight or obesity (without CVD or CKD), glucose-lowering therapy should include addon therapy with an SGLT-2 inhibitor (in those with an adequate eGFR) or a GLP-1RA with good weight loss efficacy, in addition to lifestyle management, nonsurgical energy restriction, and consideration of weight loss medications and metabolic surgery [83].

Each of the SGLT-2 inhibitors approved in the United States is indicated as an adjunct to diet and exercise to improve glycemic control in adult patients with T2D [76-79]; empagliflozin has an additional indication for reducing the risk of CV death [77] and canagliflozin is indicated to reduce the risk of MACE [76] in patients with both T2D and established CVD.

A PubMed search conducted in July 2019 identified 15 studies in which SGLT-2 inhibitors were used early in the course of T2D [64, 85-97]. Ten studies evaluated dapagliflozin [64, 85-89, 91, 93, 94, 98], two studied canagliflozin [90, 97], two assessed empagliflozin [92, 96], and one evaluated ertugliflozin [95]. Seven studies investigated SGLT-2 inhibitors as monotherapy [85-90, 98], while, in eight studies, SGLT-2 inhibitors were given in combination with metformin (Table 2) [64, 91-97]. 


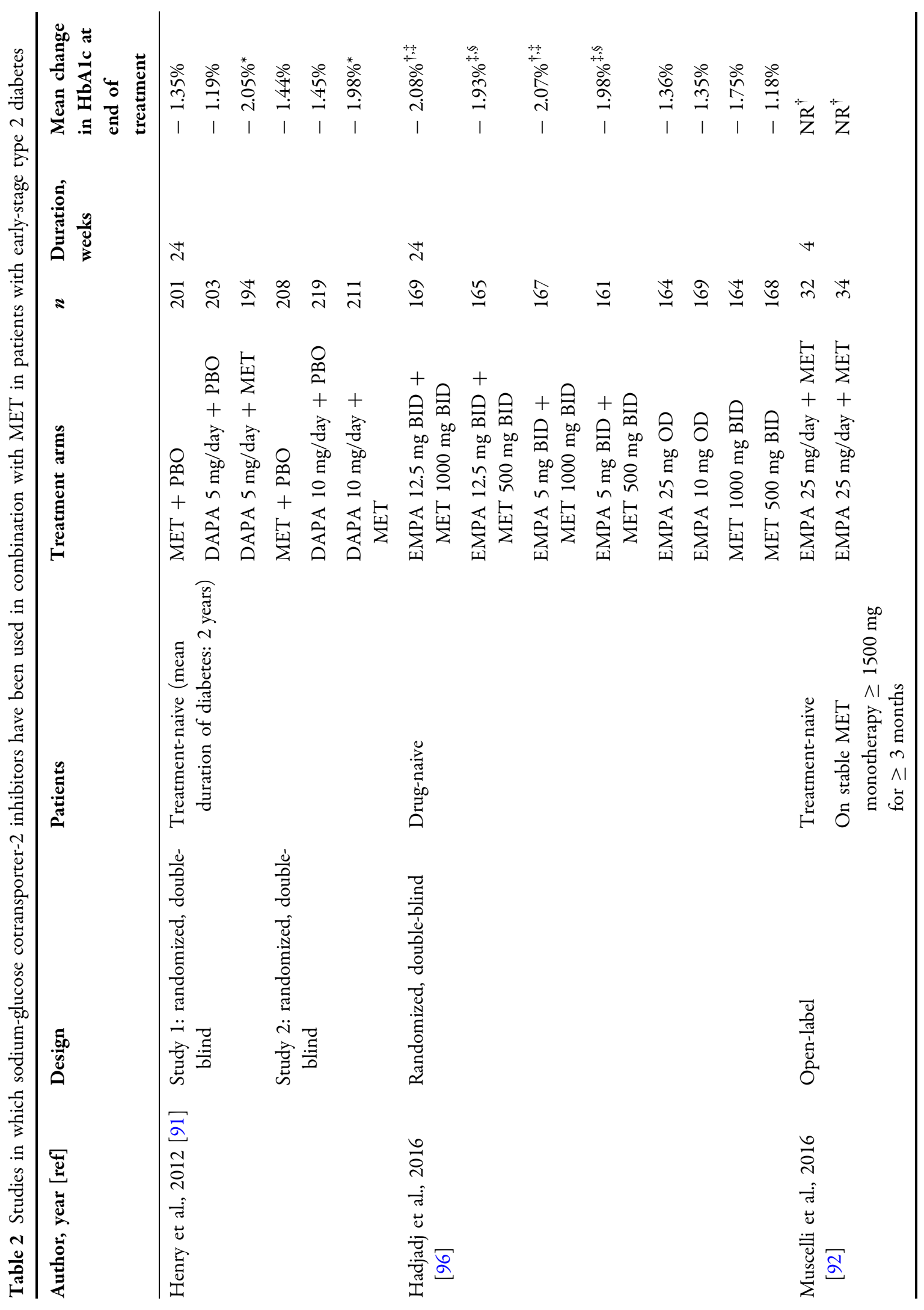




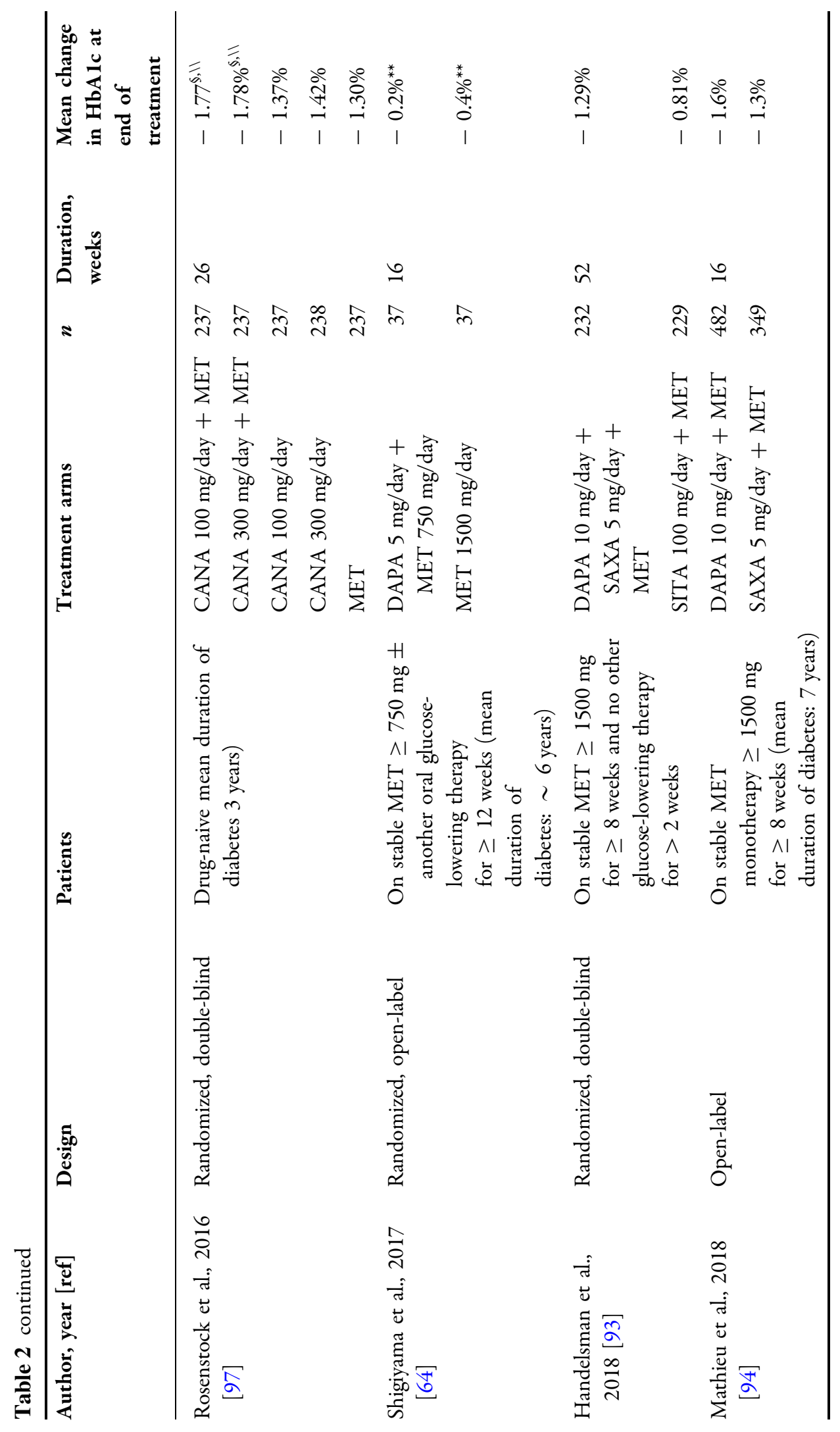




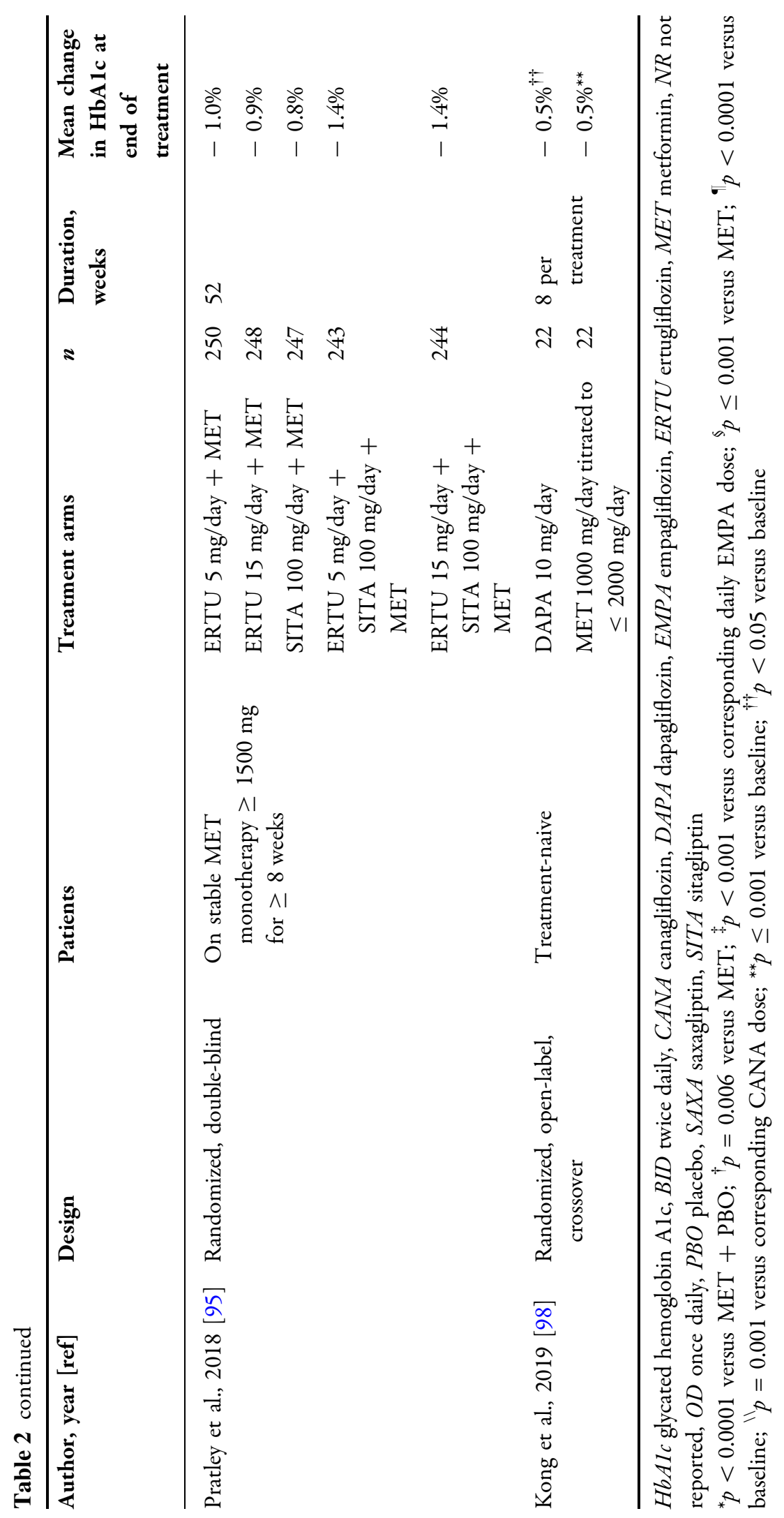


The studies that evaluated the combination of an SGLT-2 inhibitor plus metformin demonstrated a significant difference in HbA1c reduction compared with the same dose of metformin as monotherapy $[64,91,96,97]$ or compared with baseline [92]; the magnitude of the $\mathrm{HbA1c}$ reduction varied depending on study duration and background therapy at the start of treatment. Two studies have also demonstrated that the magnitude of the change in HbA1c with an SGLT-2 inhibitor, with or without metformin, was similar in treatment-naive patients as in patients who had already received metformin [92] or insulin with metformin, pioglitazone, or rosiglitazone [86]. In a study of patients with T2D receiving stable metformin therapy, early addition of dapagliflozin plus saxagliptin was associated with a significantly greater reduction in HbA1c after 26 weeks compared with addition of sitagliptin $(p=0.0008)$; the between-group difference in HbA1c reduction increased at 52 weeks [93]. Similarly, in the 52-week Evaluation of Ertugliflozin Efficacy and Safety Factorial (VERTIS FACTORIAL) study, significantly greater HbA1c reductions were observed with the combination of ertugliflozin plus sitagliptin as add-on therapy to metformin versus those with either agent individually after 26 weeks of treatment $(p<0.001)$, with sustained HbA1c reductions at 52 weeks [95].

In addition to being the guideline-recommended approach, early combination therapy in T2D makes clinical sense for several reasons [99]. First, a meta-analysis of clinical trials has demonstrated that the early use of combination therapy significantly increases the likelihood of achieving the glycemic target of HbA1c $<7 \%$ compared with metformin monotherapy [100]. Combining drugs with different mechanisms of action will have an additive effect on glycemic control while using lower doses of each drug, thereby reducing the potential for adverse events [99].

Second, the number of therapy choices to include in combination treatment is greatest early in the course of the disease when patients are relatively young and before they have developed significant comorbidities, including renal impairment, which may preclude the use of certain drugs. SGLT-2 inhibitors can be used without dose reduction in patients with mildto-moderate renal impairment [74], but, from current evidence, they are not recommended in patients with advanced kidney disease (eGFR $<45 \mathrm{~mL} / \mathrm{min} / 1.73 \mathrm{~m}^{2}$ for canagliflozin, dapagliflozin and empagliflozin and $<60 \mathrm{~mL} /$ $\mathrm{min} / 1.73 \mathrm{~m}^{2}$ for ertugliflozin) and are contraindicated in patients with severe renal impairment $\quad\left(e G F R<30 \mathrm{~mL} / \mathrm{min} / 1.73 \mathrm{~m}^{2}\right)$ [76-79]. Another reason for using SGLT-2 inhibitors early in the course of T2D is the likelihood of response to treatment, perhaps because renal function is generally better. A multivariate logistic regression analysis found that, in clinical practice, shorter T2D duration was a significant predictor of response to dapagliflozin [101]. Even among older patients (mean age at diagnosis, 57 years), early intensive glycemic control has shown benefit, with reduced risk of mortality and microvascular and macrovascular complications among those with $\mathrm{HbA} 1 \mathrm{c}<6.5 \%$ during the first year after treatment [28]. These findings underscore the potential role of SGLT-2 inhibitors in early therapy.

\section{CONCLUSIONS}

Current US treatment guidelines for T2D recommend early intensification of glucose-lowering therapy to meet glycemic targets, which usually requires the use of combination therapy $[7,8]$. Most patients with T2D have CV risk factors, such as hypertension, hypercholesterolemia, and overweight/obesity at diagnosis, so meeting and maintaining glycemic targets early in the course of T2D is the most effective way to reduce the long-term risk of complications.

SGLT-2 inhibitors appear to be an effective option for early treatment of T2D because of their favorable tolerability profile and low potential for hypoglycemia; their beneficial effects on CV risk factors, such as BP and body weight; and the evidence of benefit in terms of $\mathrm{CV}$ and renal risk reduction seen in CVOTs to date. In patients with T2D and established CVD or CKD, and those with multiple CV risk factors, SGLT-2 inhibitor therapy should be considered. 
Initiating early treatment with SGLT-2 inhibitors in patients without significant diabetesrelated complications is likely to provide the most benefit with regard to glycemic control and prevention of $\mathrm{CV}$ and renal events in patients without established CVD or CKD.

\section{ACKNOWLEDGEMENTS}

Funding. This review and the Rapid Service Fee were funded by AstraZeneca. The author had full access to the articles reviewed in this manuscript and takes complete responsibility for the integrity and accuracy of this manuscript.

Medical Writing and Editorial Assistance. Medical writing and editorial assistance was provided by Catherine Rees and Sarah Greig of inScience Communications, Springer Healthcare (Auckland, New Zealand), in accordance with Good Publication Practice (GPP-3), and was funded by AstraZeneca.

Authorship. The author meets the International Committee of Medical Journal Editors (ICMJE) criteria for authorship for this article and takes responsibility for the integrity of the work as a whole, and has given his approval for this version to be published.

Disclosures. Yehuda Handelsman has received research grants and consultant and speaker honoraria from Aegerion, Amarin, Amgen, AstraZeneca, Bristol-Myers Squibb, Boehringer Ingelheim, Eli Lilly, Gan and Lee Pharmaceuticals, Gilead Sciences, Inc., Hamni Pharmaceutical Co., Ltd., Intarcia Therapeutics, Janssen, Lexicon Pharmaceuticals, Merck \& Co., Mylan, Novo Nordisk, Pfizer, Regeneron, Sanofi, and Target PharmaSolutions.

Compliance with Ethics Guidelines. This article is based on previously conducted studies and does not contain any studies with human participants or animals performed by the author.
Data Availability. Data sharing is not applicable to this article as no datasets were generated or analyzed.

Open Access. This article is distributed under the terms of the Creative Commons Attribution-NonCommercial 4.0 International License (http://creativecommons.org/licenses/ by-nc/4.0/), which permits any noncommercial use, distribution, and reproduction in any medium, provided you give appropriate credit to the original author(s) and the source, provide a link to the Creative Commons license, and indicate if changes were made.

\section{REFERENCES}

1. Centers for Disease Control and Prevention. National Diabetes Statistics Report, 2017: Estimates of diabetes and its burden in the United States. National Center for Chronic Disease Prevention and Health Promotion; 2017. https://www.cdc.gov/ diabetes/pdfs/data/statistics/national-diabetes-statis tics-report.pdf. Accessed 13 Aug 2019.

2. Rowley WR, Bezold C, Arikan Y, Byrne E, Krohe S. Diabetes 2030: insights from yesterday, today, and future trends. Popul Health Manag. 2017;20:6-12.

3. American Diabetes Association. Economic costs of diabetes in the U.S. in 2017. Diabetes Care. 2018;41:917-28.

4. Gregg EW, Li Y, Wang J, et al. Changes in diabetesrelated complications in the United States, 1990-2010. N Engl J Med. 2014;370:1514-23.

5. Zhang N, Yang X, Zhu X, Zhao B, Huang T, Ji Q. Type 2 diabetes mellitus unawareness, prevalence, trends and risk factors: National Health and Nutrition Examination Survey (NHANES) 1999-2010. J Int Med Res. 2017;45:594-609.

6. American Diabetes Association. 10. Cardiovascular disease and risk management: Standards of Medical Care in Diabetes - 2019. Diabetes Care. 2019;42:S103-S23.

7. Garber AJ, Abrahamson MJ, Barzilay JI, et al. Consensus Statement by the American Association of Clinical Endocrinologists and American College of Endocrinology on the Comprehensive Type 2 Diabetes Management Algorithm-2019 executive summary. Endocr Pract. 2019;25:69-100. 
8. American Diabetes Association. 9. Pharmacologic approaches to glycemic treatment: Standards of Medical Care in Diabetes - 2019. Diabetes Care. 2019;42:S90-S102.

9. Neal B, Perkovic V, Mahaffey KW, et al. Canagliflozin and cardiovascular and renal events in type 2 diabetes. N Engl J Med. 2017;377:644-57.

10. Wiviott SD, Raz I, Bonaca MP, et al. Dapagliflozin and cardiovascular outcomes in type 2 diabetes. N Engl J Med. 2019;380:347-57.

11. Zinman B, Wanner C, Lachin JM, et al. Empagliflozin, cardiovascular outcomes, and mortality in type 2 diabetes. N Engl J Med. 2015;373:2117-28.

12. Marso SP, Bain SC, Consoli A, et al. Semaglutide and cardiovascular outcomes in patients with type 2 diabetes. N Engl J Med. 2016;375:1834-44.

13. Marso SP, Daniels GH, Brown-Frandsen K, et al. Liraglutide and cardiovascular outcomes in type 2 diabetes. N Engl J Med. 2016;375:311-22.

14. Hernandez AF, Green JB, Janmohamed S, et al. Albiglutide and cardiovascular outcomes in patients with type 2 diabetes and cardiovascular disease (harmony outcomes): a double-blind, randomised placebo-controlled trial. Lancet. 2018;392:1519-29.

15. Gerstein HC, Colhoun HM, Dagenais GR, et al. Dulaglutide and cardiovascular outcomes in type 2 diabetes (REWIND): a double-blind, randomised placebo-controlled trial. Lancet. 2019;294(10193): 121-30.

16. Holman RR, Bethel MA, Mentz RJ, et al. Effects of once-weekly exenatide on cardiovascular outcomes in type 2 diabetes. N Engl J Med. 2017;377:1228-39.

17. Mentz RJ, Bethel MA, Merrill P, et al. Effect of onceweekly exenatide on clinical outcomes according to baseline risk in patients with type 2 diabetes mellitus: insights from the EXSCEL trial. J Am Heart Assoc. 2018;7:e009304.

18. Zelniker TA, Wiviott SD, Raz I, et al. Comparison of the effects of glucagon-like peptide receptor agonists and sodium-glucose co-transporter 2 inhibitors for prevention of major adverse cardiovascular and renal outcomes in type 2 diabetes mellitus: a systematic review and meta-analysis of cardiovascular outcomes trials. Circulation. 2019;139:2022-31.

19. Holman RR, Paul SK, Bethel MA, Matthews DR, Neil HA. 10-year follow-up of intensive glucose control in type 2 diabetes. N Engl J Med. 2008;359:1577-89.

20. Patel A, MacMahon S, Chalmers J, et al. Intensive blood glucose control and vascular outcomes in patients with type 2 diabetes. $\mathrm{N}$ Engl J Med. 2008;358:2560-72.

21. Gæde P, Oellgaard J, Carstensen B, et al. Years of life gained by multifactorial intervention in patients with type 2 diabetes mellitus and microalbuminuria: 21 years follow-up on the Steno-2 randomised trial. Diabetologia. 2016;59:2298-307.

22. Gerstein HC, Miller ME, Byington RP, et al. Effects of intensive glucose lowering in type 2 diabetes. N Engl J Med. 2008;358:2545-59.

23. Duckworth W, Abraira C, Moritz T, et al. Glucose control and vascular complications in veterans with type 2 diabetes. N Engl J Med. 2009;360:129-39.

24. Skyler JS, Bergenstal R, Bonow RO, et al. Intensive glycemic control and the prevention of cardiovascular events: implications of the ACCORD, ADVANCE, and VA Diabetes Trials: a position statement of the American Diabetes Association and a Scientific Statement of the American College of Cardiology Foundation and the American Heart Association. J Am Coll Cardiol. 2009;53:298-304.

25. Riddle MC, Ambrosius WT, Brillon DJ, et al. Epidemiologic relationships between $\mathrm{A} 1 \mathrm{C}$ and all-cause mortality during a median 3.4-year follow-up of glycemic treatment in the ACCORD trial. Diabetes Care. 2010;33:983-90.

26. Duckworth WC, Abraira C, Moritz TE, et al. The duration of diabetes affects the response to intensive glucose control in type 2 subjects: the VA Diabetes Trial. J Diabetes Complicat. 2011;25:355-61.

27. Diabetes Control and Complications Trial (DCCT) and Epidemiology of Diabetes Interventions and Complications (EDIC) Study Research Group. Intensive diabetes treatment and cardiovascular outcomes in type 1 diabetes: the DCCT/EDIC study 30-year follow-up. Diabetes Care. 2016;39:686-93.

28. Laiteerapong N, Ham SA, Gao Y, et al. The legacy effect in type 2 diabetes: impact of early glycemic control on future complications (the diabetes and aging study). Diabetes Care. 2019;42:416-26.

29. Abdul-Ghani MA, Puckett C, Triplitt C, et al. Initial combination therapy with metformin, pioglitazone and exenatide is more effective than sequential addon therapy in subjects with new-onset diabetes. Results from the Efficacy and Durability of Initial Combination Therapy for Type 2 Diabetes (EDICT): a randomized trial. Diabetes Obes Metab. 2015;17:268-75.

30. Abdul-Ghani M, Puckett CL, Triplitt CL, et al. Durable HbA1c reduction with initial combination therapy with metformin/pioglitazone/exenatide in 
subjects with new-onset diabetes-six-year followup of the EDICT study. Diabetes. 2018;67.

31. Abdul-Ghani MA, DeFronzo RA, Norton L. Novel hypothesis to explain why SGLT2 inhibitors inhibit only $30-50 \%$ of filtered glucose load in humans. Diabetes. 2013;62:3324-8.

32. Vallon V, Thomson SC. Targeting renal glucose reabsorption to treat hyperglycaemia: the pleiotropic effects of SGLT2 inhibition. Diabetologia. 2017;60:215-25.

33. Staels B. Cardiovascular protection by sodium glucose cotransporter 2 inhibitors: potential mechanisms. Am J Med. 2017;130:S30-9.

34. Zelniker TA, Braunwald E. Cardiac and renal effects of sodium-glucose co-transporter 2 inhibitors in diabetes: JACC state-of-the-art review. J Am Coll Cardiol. 2018;72:1845-55.

35. Matsubayashi Y, Nojima T, Yoshida A, et al. Influence of SGLT2 inhibitor on resting heart rate (RHR) and factors related to its changes. Diabetes. 2018;67(suppl 1):Abstract 1154-P.

36. Kuchay MS, Krishan S, Mishra SK, et al. Effect of empagliflozin on liver fat in patients with type 2 diabetes and nonalcoholic fatty liver disease: a randomized controlled trial (E-LIFT trial). Diabetes Care. 2018;41:1801-8.

37. Raj H, Durgia H, Palui R, et al. SGLT-2 inhibitors in non-alcoholic fatty liver disease patients with type 2 diabetes mellitus: a systematic review. World J Diabetes. 2019;10:114-32.

38. Akuta N, Kawamura Y, Watanabe C, et al. Impact of sodium glucose cotransporter 2 inhibitor on histological features and glucose metabolism of non-alcoholic fatty liver disease complicated by diabetes mellitus. Hepatol Res. 2019;49:531-9.

39. Itani T, Ishihara T. Efficacy of canagliflozin against nonalcoholic fatty liver disease: a prospective cohort study. Obes Sci Pract. 2018;4:477-82.

40. Bouchi R, Terashima M, Sasahara Y, et al. Luseogliflozin reduces epicardial fat accumulation in patients with type 2 diabetes: a pilot study. Cardiovasc Diabetol. 2017;16:32.

41. Kohan DE, Fioretto P, Tang W, List JF. Long-term study of patients with type 2 diabetes and moderate renal impairment shows that dapagliflozin reduces weight and blood pressure but does not improve glycemic control. Kidney Int. 2014;85:962-71.

42. Kosiborod M, Cavender MA, Fu AZ, et al. Lower risk of heart failure and death in patients initiated on sodium-glucose cotransporter-2 inhibitors versus other glucose-lowering drugs: the CVD-REAL Study (Comparative Effectiveness of Cardiovascular Outcomes in New Users of Sodium-Glucose Cotransporter-2 Inhibitors). Circulation. 2017;136:249-59.

43. Birkeland KI, Jorgensen ME, Carstensen B, et al. Cardiovascular mortality and morbidity in patients with type 2 diabetes following initiation of sodiumglucose co-transporter-2 inhibitors versus other glucose-lowering drugs (CVD-REAL Nordic): a multinational observational analysis. Lancet Diabetes Endocrinol. 2017;5:709-17.

44. Kosiborod M, Lam CSP, Kohsaka S, et al. Cardiovascular events associated with SGLT-2 inhibitors versus other glucose-lowering drugs: the CVD-real 2 study. J Am Coll Cardiol. 2018;71:2628-39.

45. Cannon CP, McGuire DK, Pratley R, et al. Design and baseline characteristics of the eValuation of ERTugliflozin effIcacy and Safety CardioVascular outcomes trial (VERTIS-CV). Am Heart J. 2018;206:11-23.

46. Furtado RHM, Bonaca MP, Raz I, et al. Dapagliflozin and cardiovascular outcomes in patients with type 2 diabetes and prior myocardial infarction: a subanalysis from the DECLARE TIMI-58 trial. Circulation. 2019;139:2516-27.

47. Zelniker TA, Wiviott SD, Raz I, et al. SGLT2 inhibitors for primary and secondary prevention of cardiovascular and renal outcomes in type 2 diabetes: a systematic review and meta-analysis of cardiovascular outcome trials. Lancet. 2019;393:31-9.

48. Mahaffey KW, Neal B, Perkovic V, et al. Canagliflozin for primary and secondary prevention of cardiovascular events: results from the CANVAS program (Canagliflozin Cardiovascular Assessment Study). Circulation. 2018;137:323-34.

49. Wanner C, Inzucchi SE, Lachin JM, et al. Empagliflozin and progression of kidney disease in type 2 diabetes. N Engl J Med. 2016;375:323-34.

50. Mosenzon O, Wiviott SD, Cahn A, et al. Effects of dapagliflozin on development and progression of kidney disease in patients with type 2 diabetes: an analysis from the DECLARE-TIMI 58 randomised trial. Lancet Diabetes Endocrinol. 2019;7:606-17.

51. Pollock C, Stefansson B, Reyner D, et al. Albuminuria-lowering effect of dapagliflozin alone and in combination with saxagliptin and effect of dapagliflozin and saxagliptin on glycaemic control in patients with type 2 diabetes and chronic kidney disease (DELIGHT): a randomised, double-blind, placebo-controlled trial. Lancet Diabetes Endocrinol. 2019;7:429-41. 
52. Jardine MJ, Mahaffey KW, Neal B, et al. The canagliflozin and renal endpoints in diabetes with established nephropathy clinical evaluation (CREDENCE) study rationale, design, and baseline characteristics. Am J Nephrol. 2017;46:462-72.

53. Perkovic V, Jardine MJ, Neal B, et al. Canagliflozin and renal outcomes in type 2 diabetes and nephropathy N Engl J Med. 2019;380:2295-306.

54. Roche N, Reddel HK, Agusti A, et al. Integrating real-life studies in the global therapeutic research framework. Lancet Respir Med. 2013;1:e29-30.

55. Silverman SL. From randomized controlled trials to observational studies. Am J Med. 2009;122:114-20.

56. Ryan PB, Buse JB, Schuemie MJ, et al. Comparative effectiveness of canagliflozin, SGLT2 inhibitors and non-SGLT2 inhibitors on the risk of hospitalization for heart failure and amputation in patients with type 2 diabetes mellitus: a real-world meta-analysis of 4 observational databases (OBSERVE-4D). Diabetes Obes Metab. 2018;20:2585-97.

57. Patorno E, Pawar A, Franklin JM, et al. Empagliflozin and the risk of heart failure hospitalization in routine clinical care: a first analysis from the Empagliflozin Comparative Effectiveness and Safety (EMPRISE) study. Circulation. 2019;139:2822-30.

58. Mazidi M, Rezaie P, Gao HK, Kengne AP. Effect of sodium-glucose cotransport-2 inhibitors on blood pressure in people with type 2 diabetes mellitus: a systematic review and meta-analysis of 43 randomized control trials with 22528 patients. J Am Heart Assoc. 2017;6:pii: e004007.

59. Wanner C. EMPA-REG OUTCOME: the Nephrologist's point of view. Am J Med. 2017;130:S63-72.

60. Matsutani D, Sakamoto M, Kayama Y, Takeda N, Horiuchi R, Utsunomiya K. Effect of canagliflozin on left ventricular diastolic function in patients with type 2 diabetes. Cardiovasc Diabetol. 2018;17:73.

61. Verma S, Garg A, Yan AT, et al. Effect of empagliflozin on left ventricular mass and diastolic function in individuals with diabetes: an important clue to the EMPA-REG OUTCOME trial? Diabetes Care. 2016;39:e212-3.

62. Kubota Y, Yamamoto T, Tara S, et al. Effect of empagliflozin versus placebo on cardiac sympathetic activity in acute myocardial infarction patients with type 2 diabetes mellitus: rationale. Diabetes Ther. 2018;9:2107-16.

63. Lytvyn Y, Bjornstad P, Udell JA, Lovshin JA, Cherney DZI. Sodium glucose cotransporter-2 inhibition in heart failure: potential mechanisms, clinical applications, and summary of clinical trials. Circulation. 2017;136:1643-58.

64. Shigiyama F, Kumashiro N, Miyagi M, et al. Effectiveness of dapagliflozin on vascular endothelial function and glycemic control in patients with early-stage type 2 diabetes mellitus: DEFENCE study. Cardiovasc Diabetol. 2017;16:84.

65. Ferrannini E, Baldi S, Frascerra S, et al. Shift to fatty substrate utilization in response to sodium-glucose cotransporter 2 inhibition in subjects without diabetes and patients with type 2 diabetes. Diabetes. 2016;65:1190-5.

66. Mudaliar S, Alloju S, Henry RR. Can a shift in fuel energetics explain the beneficial cardiorenal outcomes in the EMPA-REG OUTCOME study? A unifying hypothesis. Diabetes Care. 2016;39:1115-22.

67. Packer M, Anker SD, Butler J, Filippatos G, Zannad F. Effects of sodium-glucose cotransporter 2 inhibitors for the treatment of patients with heart failure: proposal of a novel mechanism of action. JAMA Cardiol. 2017;2:1025-9.

68. Sato T, Aizawa Y, Yuasa S, et al. The effect of dapagliflozin treatment on epicardial adipose tissue volume. Cardiovasc Diabetol. 2018;17:6.

69. Verma S, McMurray JJV. SGLT2 inhibitors and mechanisms of cardiovascular benefit: a state-ofthe-art review. Diabetologia. 2018;61:2108-17.

70. Lee TM, Chang NC, Lin SZ. Dapagliflozin, a selective SGLT2 inhibitor, attenuated cardiac fibrosis by regulating the macrophage polarization via STAT3 signaling in infarcted rat hearts. Free Radic Biol Med. 2017;104:298-310.

71. Lin B, Koibuchi N, Hasegawa Y, et al. Glycemic control with empagliflozin, a novel selective SGLT2 inhibitor, ameliorates cardiovascular injury and cognitive dysfunction in obese and type 2 diabetic mice. Cardiovasc Diabetol. 2014;13:148.

72. Kang S, Verma S, Teng G, et al. Direct effects of empagliflozin on extracellular matrix remodeling in human cardiac fibroblasts: novel translational clues to EMPA-REG Outcome. Can J Cardiol. 2017;33:S169.

73. Kawanami D, Matoba K, Takeda Y, et al. SGLT2 inhibitors as a therapeutic option for diabetic nephropathy. Int J Mol Sci. 2017;18:pii: E1083.

74. Handelsman Y. Potential place of SGLT2 inhibitors in treatment paradigms for type 2 diabetes mellitus. Endocr Pract. 2015;21:1054-65.

75. van Baar MJB, van Ruiten CC, Muskiet MHA, van Bloemendaal L, Ijzerman RG, van Raalte DH. SGLT2 
inhibitors in combination therapy: from mechanisms to clinical considerations in type 2 diabetes management. Diabetes Care. 2018;41:1543-56.

76. US Food and Drug Administration. Invokana ${ }^{\circledR}$ (canagliflozin) tablets, for oral use: prescribing information. 2018. https://www.accessdata.fda.gov/ drugsatfda_docs/label/2013/204042s000lbl.pdf. Accessed 13 Aug 2019.

77. US Food and Drug Administration. Jardiance ${ }^{\circledR}$ (empagliflozin) tablets, for oral use: prescribing information. 2018. https://www.accessdata.fda.gov/ drugsatfda_docs/label/2016/204629s008lbl.pdf. Accessed 13 Aug 2019.

78. US Food and Drug Administration. Steglatro ${ }^{\mathrm{TM}}$ (ertugliflozin) tablets, for oral use: prescribing information. 2018. https://www.accessdata.fda.gov/ drugsatfda_docs/label/2017/209803s000lbl.pdf. Accessed 13 Aug 2019.

79. US Food and Drug Administration. Farxiga ${ }^{\circledR}$ (dapagliflozin) tablets, for oral use: prescribing information. 2019. https://www.accessdata.fda.gov/drug satfda_docs/label/2014/202293s000lbl.pdf. Accessed 13 Aug 2019.

80. Handelsman Y, Henry RR, Bloomgarden ZT, et al. American Association of Clinical Endocrinologists and American College of Endocrinology Position Statement on the Association of Sglt-2 Inhibitors and Diabetic Ketoacidosis. Endocr Pract. 2016;22:753-62.

81. Ueda P, Svanstrom H, Melbye M, et al. Sodium glucose cotransporter 2 inhibitors and risk of serious adverse events: nationwide register based cohort study. BMJ. 2018;363:k4365.

82. Zhang XL, Zhu QQ, Chen YH, et al. Cardiovascular safety, long-term noncardiovascular safety, and efficacy of sodium-glucose cotransporter 2 inhibitors in patients with type 2 diabetes mellitus: a systemic review and meta-analysis with trial sequential analysis. J Am Heart Assoc. 2018;7(2).

83. Davies MJ, D'Alessio DA, Fradkin J, Management of hyperglycemia in type 2 diabetes, et al. A consensus report by the American Diabetes Association (ADA) and the European Association for the Study of Diabetes (EASD). Diabetes Care. 2018;2018(41):2669-701.

84. Arnett DK, Blumenthal RS, Albert MA, et al. 2019 ACC/AHA guideline on the primary prevention of cardiovascular disease: a report of the American College of Cardiology/American Heart Association Task Force on Clinical Practice Guidelines. J Am Coll Cardiol. 2019. https://doi.org/10.1016/j.jacc. 2019.03.010.
85. Ferrannini E, Ramos SJ, Salsali A, Tang W, List JF. Dapagliflozin monotherapy in type 2 diabetic patients with inadequate glycemic control by diet and exercise: a randomized, double-blind, placebocontrolled, phase 3 trial. Diabetes Care. 2010;33:2217-24.

86. Zhang L, Feng Y, List J, Kasichayanula S, Pfister M. Dapagliflozin treatment in patients with different stages of type 2 diabetes mellitus: effects on glycaemic control and body weight. Diabetes Obes Metab. 2010;12:510-6.

87. Bailey CJ, Iqbal N, T'Joen C, List JF. Dapagliflozin monotherapy in drug-naive patients with diabetes: a randomized-controlled trial of low-dose range. Diabetes Obes Metab. 2012;14:951-9.

88. Bailey CJ, Morales Villegas EC, Woo V, Tang W, Ptaszynska A, List JF. Efficacy and safety of dapagliflozin monotherapy in people with type 2 diabetes: a randomized double-blind placebocontrolled 102-week trial. Diabetes Med. 2015;32:531-41.

89. Li FF, Gao G, Li Q, et al. Influence of dapagliflozin on glycemic variations in patients with newly diagnosed type 2 diabetes mellitus. J Diabetes Res. 2016;2016:5347262.

90. Kutoh E, Wada A, Murayama T, Takizawa Y. Canagliflozin as an initial therapy in drug-naive subjects with type 2 diabetes mellitus: a potential involvement of atherogenic lipids in its glycemic efficacy. Drugs R D. 2017;17:313-20.

91. Henry RR, Murray AV, Marmolejo MH, Hennicken D, Ptaszynska A, List JF. Dapagliflozin, metformin $\mathrm{XR}$, or both: initial pharmacotherapy for type 2 diabetes, a randomised controlled trial. Int J Clin Pract. 2012;66:446-56.

92. Muscelli E, Astiarraga B, Barsotti E, et al. Metabolic consequences of acute and chronic empagliflozin administration in treatment-naive and metformin pretreated patients with type 2 diabetes. Diabetologia. 2016;59:700-8.

93. Handelsman Y, Mathieu C, Del Prato S, et al. Sustained 52-week efficacy and safety of triple therapy with dapagliflozin plus saxagliptin versus dual therapy with sitagliptin added to metformin in patients with uncontrolled type 2 diabetes. Diabetes Obes Metab. 2019;21:883-92.

94. Mathieu C, Catrinoiu D, Ranetti AE, et al. Characterization of the open-label lead-in period of two randomized controlled phase 3 trials evaluating dapagliflozin, saxagliptin, and metformin in type 2 diabetes. Diabetes Ther. 2018;9:1703-11. 
95. Pratley RE, Eldor R, Raji A, et al. Ertugliflozin plus sitagliptin versus either individual agent over 52 weeks in patients with type 2 diabetes mellitus inadequately controlled with metformin: the VERTIS FACTORIAL randomized trial. Diabetes Obes Metab. 2018;20:1111-20.

96. Hadjadj S, Rosenstock J, Meinicke T, Woerle HJ, Broedl UC. Initial combination of empagliflozin and metformin in patients with type 2 diabetes. Diabetes Care. 2016;39:1718-28.

97. Rosenstock J, Chuck L, Gonzalez-Ortiz M, et al. Initial combination therapy with canagliflozin plus metformin versus each component as monotherapy for drug-naive type 2 diabetes. Diabetes Care. 2016;39:353-62.
98. Kong SH, Koo BK, Moon MK. Effects of dapagliflozin on endothelial function, renal injury markers, and glycemic control in drug-naive patients with type 2 diabetes mellitus. Diabetes Metab J. 2019. https:// doi.org/10.4093/dmj.2018.0208.

99. Handelsman Y, Jellinger PS. Overcoming obstacles in risk factor management in type 2 diabetes mellitus. J Clin Hypertens. 2011;13:613-20.

100. Phung OJ, Sobieraj DM, Engel SS, Rajpathak SN. Early combination therapy for the treatment of type 2 diabetes mellitus: systematic review and metaanalysis. Diabetes Obes Metab. 2014;16:410-7.

101. Han E, Kim A, Lee SJ, et al. Characteristics of dapagliflozin responders: a longitudinal, prospective, nationwide dapagliflozin surveillance study in Korea. Diabetes Ther. 2018;9:1689-701. 